\title{
Nonlinear Vibrations of a Rotor-Fluid-Foundation System Supported by Rolling Bearings
}

\author{
Almatbek Kydyrbekuly ${ }^{1}$ - Leyla Khajiyeva ${ }^{1}$ - Alysher Ybraev Gulama-Garyp ${ }^{1}$ - Julius Kaplunov ${ }^{2}$ \\ 1Al-Farabi Kazakh National University, Kazakhstan \\ 2Keele University, United Kingdom
}

A generalized dynamic model taking into account coupled vibrations of a rotor-fluid-foundation system, linear eccentricity, damping, and rolling bearing nonlinearity is developed. The nonlinear equations of motion are formulated and analysed. Forced and free vibrations of the system are investigated. Peculiarities of the dynamic behaviour are revealed, including properties of vibration frequencies and amplitudes. The obtained results have the potential to be implemented in the optimal design of modern industrial devices.

Keywords: rotor, foundation, rolling bearing, nonlinear vibration, resonance, fluid, cavity

Highlights

- A non-linear mathematical model for a rotor with a fluid filled cavity is developed.

- The equations of motion for a 'rotor-fluid-foundation' system are derived and solved.

- Simple explicit formulae for analysing eigen and forced vibrations are obtained.

- Conditions on problem parameters corresponding to the minimal pressure on supports and self-centring are presented.

\section{INTRODUCTION}

High-speed rotating machines are widely spread in modern technology. Rotors and shafts are key components responsible for transmission of torque and rotation in the majority of power, electric and drilling systems [1] and [2]. In particular, these include gas turbines, compressors, separators, centrifugal pumps and textile machines, e.g. see [2] and [3].

Systems with fluid-filled cavities are an important class of rotating machines. Among them are elastic fluid turbines, fluid gyroscopes, centrifuges, and separators. A rotor with fluid-filled cavities is a non-conservative mechanical system [4] due to selfoscillation caused by the presence of fluid [5] and [6]. At supercritical speeds fluid converts rotor rotation energy into its transverse vibration energy resulting in instability [7] and [8]. Analysis of experimental data shows that fluid in rotor cavities is often the main cause of unstable regimes [9] and [10]. The underlying physics is that the fluid centrifugal acceleration is opposite to the pressure gradient leading to violation of equilibrium [11] and [12].

In addition to the above mentioned role of fluid, rotor dynamics is also affected by a few other parameters, e.g. see [13] and [14], including the variation of inertial characteristics, nonlinearity and stiffness of supports and shafts, rotor disbalance and asymmetry [15], specific properties of a lubrication layer in case of plain bearings [16] and, especially, performance of rolling bearings [17] and [18], internal and external damping, and some others.

Modern design and calculation of the vibration characteristics of rotating machines require treatment of the whole mechanical "rotor-fluid-foundation" system. Many theoretical and applied considerations on the subject concentrate on vibration of rotor and fluid only, neglecting foundation motion. Such assumption may considerably affect the accuracy of evaluation of the overall dynamic and kinematic behaviour of the system [19]. Experimental analysis clearly indicates the importance of taking into account foundation vibration along with the development of methods for its reduction [20] and [21].

Nowadays, the rotating machines widely used in industry mostly operate with rolling bearings [22] and [23]. Although plain bearings with liquid or gas lubrication have some advantages in comparison with rolling bearings, they have not yet found major industrial applications. The reason is that a lubrication layer induces high-amplitude self-oscillation in the system leading to a quick bearing failure [16] and [24]. In this case stresses in bearings are the result of the pressure in liquid films [25].

The increase in rotation speeds has natural restrictions due to the mechanical properties of rotating machines. At relatively low speeds design consists, as a rule, sufficient balancing. Ideally, dynamic stresses between the rotor and bearing supports and consequently induced vibrations disappear provided that the rotor principal inertia axis coincides with its 
axis of rotation. In this case, some of the important bearing properties, including nonlinear deformability, are ignored [18]. However, at higher speeds the effect of bearing nonlinear deformability becomes essential.

Rotor dynamics in the case of nonlinear elastic supports, e.g. rolling bearings, has not been fully studied. A rolling bearing can be modelled as a rigid body support which is a hinge for a single row ball bearing and a clamped end for a support equipped with a pair of double row bearings. This simplified approach, however, does not produce a sufficiently accurate solution of the original engineering problem. The practice needs more general mathematical models incorporating far more specific features of rolling bearings, such as geometric uncertainties and gaps as well as their variation at exploitation [26] and [27], nonlinear stiffnesses [28] and [29], centrifugal forces, sophisticated behaviour of bearing rings, gyroscopic phenomena [30], friction in lubrication layers, variable thickness of lubrication layers along contact surfaces [16], etc.

In this paper, we investigate the nonlinear dynamics of a rotor with a fluid-filled cavity. The rotor is assumed to be supplied with rotation bearings and elastically supported by a foundation. The studied system models vertical centrifuges widely used for separation of various heterogeneous mixtures. The increase in rotation speeds along with the demand for a high precision performance motivates advanced modelling taking into account the deformability of rolling bearings. Their nonlinear behaviour seems to be essential for rotor dynamics. In particular, radial compression arises from deformations of rolling bodies along contact surfaces.

The challenge of the problem in question is also due to a strong coupling between the rotor and fluid motions affecting vibration frequencies and causing instability. The equations analysed in the paper govern rigid body fluid interaction. High-speed rotor modelling assumes evaluating the influence of the phenomena mentioned above in order to arrive at the most optimal problem formulation by preserving only its key features.

\section{STATEMENT OF THE PROBLEM}

Consider a symmetric vertical rotor of mass $m$ with a cylindrical cavity of radius $R$ partially filled with an ideal fluid, see Fig. 1. The angular speed of the rotor $\Omega_{0}=$ const is considered to be greater than the associated critical speed. Let the rotor together with fluid perform rigid body rotation under dynamic equilibrium. We also assume static imbalance of the rotor, denoting it by $e$. The analysed system rests on rolling bearings. Elastic deformations arising in bearings are supposed to have both radial and axial components, demonstrating essential nonlinearity. The radial compliance of bearings is considered to occur due to the deformability of rolling elements and raceways along contact zones.

The equations of static equilibrium of a bearing may be derived from the Hertz theory. The nonlinear bearing stiffness is taken as $K(\delta)=c_{0} \delta+c_{1} \delta^{3}$, where $\delta$ is the minimal distance between the inner and outer rings of the bearing. The restoring force in radial bearings is obtained by a power series approximation. The outer ring is attached to a foundation of mass $M$ resting on an elastic support with a linear stiffness coefficient $c_{2}$.

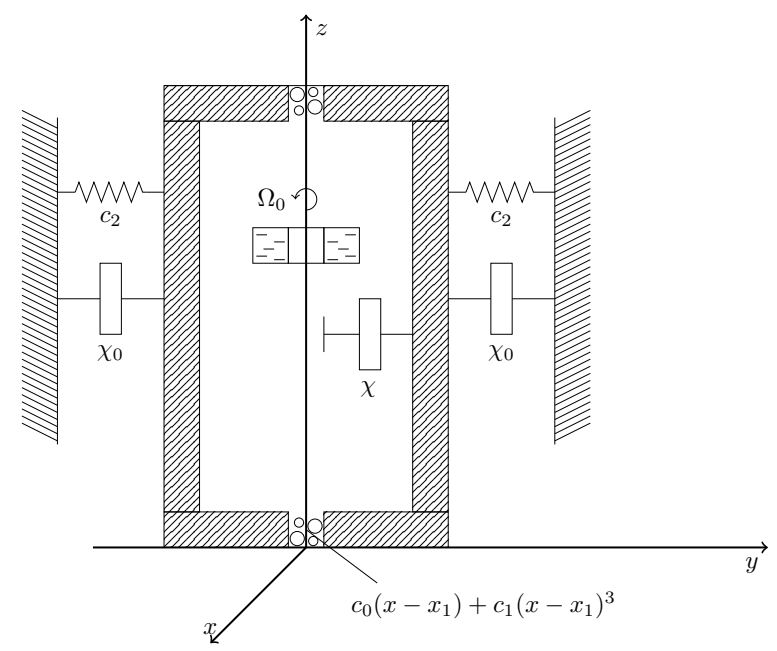

Fig. 1. Rotor with rolling bearings resting on an elastic foundation

Specify a Cartesian coordinate system Oxyz assuming that, in equilibrium, the geometric centre of the rotor and the centre of mass of the foundation both lie on the $O z$ axis. Below $x$ and $y$ are the coordinates of the rotor center, while $x_{1}$ and $y_{1}$ are the coordinates of the center mass of the foundation. We also use the notations $c_{0}$ and $c_{1}$ for rolling bearing stiffnesses, and $\chi$ and $\chi_{0}$ stand for the coefficients of external damping.

We suppose that the rotor performs a planeparallel motion, and the foundation has no rotation around coordinate axes. In this case, the equations of motion can be written as: 


$$
\begin{gathered}
m \ddot{x}+2 c_{0}\left(x-x_{1}\right)+2 c_{1}\left(x-x_{1}\right)^{3}+\chi \dot{x}= \\
=m e \Omega_{0}^{2} \cos \Omega_{0} t+F_{x}, \\
m \ddot{y}+2 c_{0}\left(y-y_{1}\right)+2 c_{1}\left(y-y_{1}\right)^{3}+\chi \dot{y}= \\
=m e \Omega_{0}^{2} \sin \Omega_{0} t+F_{y}, \quad \text { and } \\
M \dot{x}_{1}+2 c_{2} x_{1}-2 c_{0}\left(x-x_{1}\right)-2 c_{1}\left(x-x_{1}\right)^{3}+\chi_{0} \dot{x}_{1}=0, \\
M \dot{y}_{1}+2 c_{2} y_{1}-2 c_{0}\left(y-y_{1}\right)-2 c_{1}\left(y-y_{1}\right)^{3}+\chi_{0} \dot{y}_{1}=0,
\end{gathered}
$$

where $F_{x}$ and $F_{y}$ are the components of the fluid reaction force given by:

$$
\begin{aligned}
& F_{x}=\left.R h \int_{0}^{2 \pi} P\right|_{r=R} \cos \left(\Omega_{0} t+\varphi\right) d \varphi, \\
& F_{y}=\left.R h \int_{0}^{2 \pi} P\right|_{r=R} \sin \left(\Omega_{0} t+\varphi\right) d \varphi .
\end{aligned}
$$

Here $h$ is the height of the cavity and $P$ is the fluid pressure along the wall of the rotor.

The equations of fluid motion can be written as, e.g. see [6],

$$
\begin{gathered}
\frac{\partial u}{\partial t}-2 \Omega_{0} v= \\
=-\frac{1}{\rho} \frac{\partial P}{\partial r}-\ddot{x} \cos \left(\Omega_{0} t+\varphi\right)-\ddot{y} \sin \left(\Omega_{0} t+\varphi\right), \\
\frac{\partial v}{\partial t}+2 \Omega_{0} u= \\
=-\frac{1}{\rho r} \frac{\partial P}{\partial \varphi}+\ddot{x} \sin \left(\Omega_{0} t+\varphi\right)-\ddot{y} \sin \left(\Omega_{0} t+\varphi\right) .
\end{gathered}
$$

The continuity equation at $\rho=$ const is:

$$
\frac{\partial(u r)}{\partial r}+\frac{\partial v}{\partial \varphi}=0 .
$$

The boundary conditions take the form: the impenetrability condition:

$$
\left.u\right|_{r=R}=0,
$$

the condition on a free fluid surface:

$$
\left.P\right|_{r=r_{0}}=0 \quad \text { or } \quad \frac{\partial P}{\partial t}=-\left.\rho \Omega_{0}{ }^{2} r_{0} u\right|_{r=r_{0}} .
$$

In the formulae above, $u$ and $v$ are radial and circumferential components of fluid velocity, $\rho$ is fluid density, $r_{0}$ is the free surface radius, $r$ and $\varphi$ are cylindrical polars.

\section{SOLUTION OF EQUATIONS OF MOTION}

First, solve the equations of fluid motion Eqs. (4) to (7) using the stream function $\Phi$ and the velocity potential $\psi$. In this case, the components of fluid velocity become:

$$
u=\frac{\partial \Phi}{\partial r}, v=\frac{1}{r} \frac{\partial \Phi}{\partial \varphi} \quad \text { or } \quad u=\frac{1}{r} \frac{\partial \psi}{\partial \phi}, v=-\frac{\partial \psi}{\partial r} .
$$

Then, on introducing the expressions for $u$ and $v$ from Eq. (8) into the Eq. (4), we obtain:

$$
\begin{aligned}
\operatorname{grad}\left\{\frac{\partial \$}{\partial t}\right. & +2 \Omega_{0} \psi+\frac{P}{\rho}+r\left[\ddot{x} \cos \left(\Omega_{0} t+\phi\right)+\right. \\
& \left.\left.+\ddot{y} \sin \left(\Omega_{0} t+\phi\right)\right]\right\}=0 .
\end{aligned}
$$

Furthermore, the continuity equation can be rewritten as:

$$
\Delta \Phi=0,
$$

where $\Delta$ is the Laplace operator specified in cylindrical polar coordinates.

From Eq. (9), we determine the expression for fluid pressure as:

$$
\begin{gathered}
P=-\rho\left\{\frac{\partial \Phi}{\partial t}+2 \Omega_{0} \psi+r\left[\ddot{x} \cos \left(\Omega_{0} t+\phi\right)+\right.\right. \\
\left.\left.+\ddot{y} \sin \left(\Omega_{0} t+\phi\right)\right]\right\} .
\end{gathered}
$$

In terms of the complex variables:

$$
x+i y=z \text {, and } x_{1}+i y_{1}=z_{1} .
$$

Eq. (11) takes the form:

$$
P=-\rho\left[\frac{\partial \Phi}{\partial t}+2 \Omega_{0} \psi+\ddot{z} r \exp \left(-i\left(\Omega_{0} t+\varphi\right)\right)\right] \text {. }
$$

In the case of time-harmonic vibrations of the rotor and foundation, the complex variables $z$ and $z_{1}$ can be presented as, see [4] to [12],

$$
\begin{gathered}
z=A \exp \left(i \Omega_{0} t\right)+B \exp (i \omega t), \\
z_{1}=C \exp \left(i \Omega_{0} t\right)+D \exp (i \omega t),
\end{gathered}
$$

where $\omega$ is the eigenfrequency.

The harmonic function $\Phi$ and the function $\psi$ taking into account Eq. (14) become:

$$
\begin{aligned}
& \Phi=R_{2}(r) \exp (i(\sigma t-\varphi)), \\
& \psi=R_{1}(r) \exp (i(\sigma t-\varphi)),
\end{aligned}
$$

where $\sigma=\omega-\Omega_{0}$.

On substituting the last expression for the stream function $\Phi$ into Eq. (10), we obtain:

$$
R_{2}(r)=C_{1} r+\frac{C_{2}}{r} \text {. }
$$

We also get for the function $\psi$, see Eq. (8), 


$$
R_{1}(r)=i\left(C_{1} r-\frac{C_{2}}{r}\right)
$$

The constants $C_{1}$ and $C_{2}$ can be found from the boundary conditions in Eqs. (6) and (7). Then, by inserting Eq. (13) into Eqs. (2) and (3) and taking into account Eqs. (16) to (19), we obtain a formula for the complex force $F_{r}$. It is:

$$
\begin{gathered}
F_{r}=F_{x}+i F_{y}=A m_{L} \Omega_{0}{ }^{2} \exp \left(i \Omega_{0} t\right)+ \\
+B m_{L} \omega^{2} \frac{\left(\sigma^{2}-2 \Omega_{0} \sigma-\Omega_{0}{ }^{2}\right)}{\left(\gamma \sigma^{2}-2 \Omega_{0} \sigma-\Omega_{0}{ }^{2}\right)} \exp (i \omega t),
\end{gathered}
$$

with $q=\frac{R}{r_{0}}, \gamma=\frac{q^{2}+1}{q^{2}-1}$ and $m_{L}=\pi \rho R^{2} h$. Here $m_{L}$ is the mass of fluid inside the cavity, and $\gamma$ characterises the relative fluid volume.

It is clear that in Eq. (1) the first and third equations are identical to the second and fourth equations, respectively. To this end, below we restrict ourselves to two equations in $x$ and $x_{1}$ :

$$
\begin{gathered}
\ddot{x}+n_{0}{ }^{2}\left(x-x_{1}\right)+n_{1}\left(x-x_{1}\right)^{3}+2 k \dot{x}= \\
=e \Omega_{0}^{2} \cos \Omega_{0} t+\operatorname{Re}\left(\frac{F_{r}}{m}\right), \\
\ddot{x}_{1}+n_{2}{ }^{2} x_{1}-n_{01}{ }^{2}\left(x-x_{1}\right)-n_{10}\left(x-x_{1}\right)^{3}+2 k_{0} \dot{x}_{1}=0,
\end{gathered}
$$

where

$$
\begin{gathered}
n_{0}{ }^{2}=\frac{2 c_{0}}{m}, n_{1}=\frac{2 c_{1}}{m}, 2 k=\frac{\chi}{m}, n_{2}{ }^{2}=\frac{2 c_{2}}{M}, \\
n_{01}{ }^{2}=\frac{2 c_{0}}{M}=\mu n_{0}{ }^{2}, n_{10}=\frac{2 c_{1}}{M}=\mu n_{1}, 2 k_{0}=\frac{\chi_{0}}{M}, \mu=\frac{m}{M} .
\end{gathered}
$$

On substituting the real parts of Eqs. (14), (15) and (20) into Eq. (21), we obtain, similarly to the derivations in [6] and [12], a set of algebraic equations in the unknowns $A, B, C$ and $D$. Then,

$$
C=P_{0}+i P_{1}+A\left(P_{2}+i P_{3}\right) \text { and } D=\left(P_{4}+i P_{5}\right) B,
$$

with

$$
\begin{gathered}
P_{0}=\frac{\mu e \Omega_{0}{ }^{2}\left(n_{2}{ }^{2}-\Omega_{0}{ }^{2}\right)}{m_{0}}, P_{1}=-\frac{2 k_{0} \Omega_{0}{ }^{3}}{m_{0}}, \\
P_{2}=\frac{\mu\left[\left(1+\mu_{L}\right) \Omega_{0}{ }^{2}\right]\left(n_{2}{ }^{2}-\Omega_{0}{ }^{2}\right)-4 k k_{0} \Omega_{0}{ }^{2}}{m_{0}}, \\
P_{3}=-\frac{2 \mu\left[\left(1+\mu_{L}\right) \Omega_{0}{ }^{2} k_{0}+\left(n_{2}{ }^{2}-\Omega_{0}{ }^{2}\right) k\right] \Omega_{0}}{m_{0}}
\end{gathered}
$$

$$
\begin{gathered}
P_{4}=\frac{\mu \omega^{2}\left(n_{2}^{2}-\omega^{2}\right)\left(1+\mu_{L} \frac{D_{3}}{D_{4}}\right)-4 k k_{0} \omega^{2}}{m_{1}}, \text { and } \\
P_{5}=-\frac{2 \mu\left[\left(1+\mu_{L} \frac{D_{3}}{D_{4}}\right) k_{0} \omega^{2}+\left(n_{2}^{2}-\omega^{2}\right) k\right] \omega}{m_{0}} .
\end{gathered}
$$

In the above $\mu_{L}=\frac{m_{L}}{m}$ and

$$
\begin{gathered}
m_{1}=\left(n_{2}{ }^{2}-\omega^{2}\right)^{2}+4 k_{0}{ }^{2} \omega^{2}, \\
m_{0}=\left(n_{2}{ }^{2}-\Omega_{0}{ }^{2}\right)^{2}+4 k_{0}{ }^{2} \Omega_{0}{ }^{2}, \\
D_{3}=\sigma^{2}-2 \Omega_{0} \sigma-\Omega_{0}{ }^{2}, \\
D_{4}=\gamma \sigma^{2}-2 \Omega_{0} \sigma-\Omega_{0}{ }^{2}, \\
D=\left(P_{4}+i P_{5}\right) B .
\end{gathered}
$$

From the last formulae, we obtain algebraic equations for $A$ and $B$, i.e.

$$
\begin{gathered}
\frac{3 n_{10} P_{75} P_{23}}{2}\left(P_{23} A^{2}-2 P_{01} A\right)-e_{2} P_{45}+ \\
+\left(n_{01}^{2}+\frac{3 n_{10}}{2} P_{01}{ }^{2}\right) P_{75}+\frac{3 n_{10}}{4} P_{75}{ }^{3} B^{2}=0, \\
\frac{3}{4} n_{10} P_{23}{ }^{3} A^{3}-\frac{9}{4} n_{10} P_{01} P_{23}{ }^{2} A^{2}+\left[P_{23}\left(n_{01}^{2}+\frac{9}{4} n_{01} P_{01}{ }^{2}\right)-\right. \\
\left.-e_{0} P_{23}{ }^{*}\right] A-\left(e_{0}+n_{01}{ }^{2}+\frac{3}{4} n_{10} P_{01}{ }^{2}\right) P_{01}+ \\
+\frac{3}{2} P_{75}{ }^{2} n_{10}\left(P_{23} A-P_{01}\right) B^{2}=0 .
\end{gathered}
$$

Here

$$
\begin{gathered}
P_{01}=P_{0}+i P_{1}, P_{23}=1-P_{2}-i P_{3}, P_{45}=P_{4}+i P_{5}, \\
P_{75}=P_{7}-i P_{5}, P_{7}=1-P_{4}, P_{23}{ }^{*}=P_{2}+i P_{3}, \\
e_{0}=n_{2}{ }^{2}-\Omega_{0}{ }^{2}+2 i k_{0} \Omega_{0}, e_{2}=n_{2}{ }^{2}-\omega^{2}+2 k_{0} i \omega .
\end{gathered}
$$

Now, we determine $B^{2}$ from the first equation in Eq. (24) and substitute it into the second equation. As a result, we obtain a third order equation for $A$ which can be easily solved, for example, using the Cardano formula. Finally, we have the constants $C$ and $D$ from Eq. (22). The derived formulae for $A, B, C$ and $D$ are typical for nonlinear systems demonstrating a specific relation between coefficients.

The developed approach can be extended to a cavity filled with viscous fluid. It might be expected that the nonlinear elastic properties of bearings should not considerably affect the motion of a viscous fluid. In fact, the ideal fluid approximation assumed in the paper has been implemented only because this results 
in a relatively simple formulae useful for potential engineering applications.

\section{FREE NONLINEAR VIBRATIONS OF THE ROTOR WITH CAVITY PARTIALLY FILLED WITH FLUID}

Consider a rotating system neglecting imbalance and concentrating on nonlinear vibrations of a rotor with a partially fluid filled cavity resting on an elastic foundation. The equations of motion neglecting imbalance take the form:

$$
\begin{gathered}
\ddot{x}+n_{0}^{2}\left(x-x_{1}\right)+n_{1}\left(x-x_{1}\right)^{3}+2 k \dot{x}=\operatorname{Re}\left(\frac{F_{r}}{m}\right), \\
\ddot{x}_{1}+n_{2}^{2} x_{1}-n_{01}{ }^{2}\left(x-x_{1}\right)-n_{10}\left(x-x_{1}\right)^{3}+2 k_{0} \dot{x}_{1}=0,
\end{gathered}
$$

where the former notations are adapted and the force $F_{r}$ is expressed as [12]:

$$
F_{r}=B m_{L} \omega^{2} \frac{\left(\sigma^{2}-2 \Omega_{0} \sigma-\Omega_{0}^{2}\right)}{\left(\gamma \sigma^{2}-2 \Omega_{0} \sigma-\Omega_{0}^{2}\right)} \exp (i \omega t) .
$$

As above, we seek the solution in the form:

$$
\begin{aligned}
& x=B \exp (i \omega t), \\
& x_{1}=D \exp (i \omega t) .
\end{aligned}
$$

On substituting Eqs. (27) and (28) into Eq. (25) and using the same procedure as in the previous section, we obtain:

$$
D=P_{8} B
$$

where $P_{8}=P_{9}+i P_{10}$,

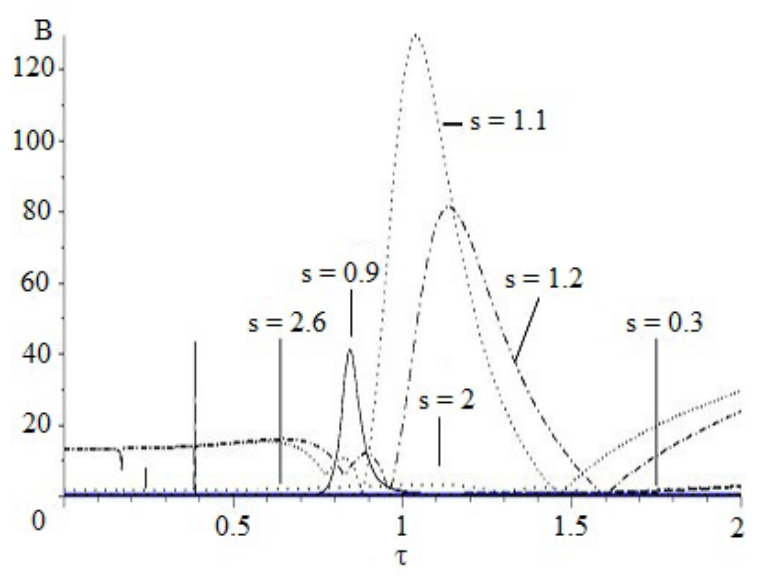

Fig. 2. Rotor amplitude $B=B(\tau, S)$ at $\gamma=15.24$, taking into account damping

$$
\begin{aligned}
& \text { with } P_{9}=\frac{\mu\left[\tau^{2} D_{6}\left(n_{02}{ }^{2}-\tau^{2}\right)-4 k_{01} \tau^{2}\right]}{g_{0}}, \\
& \text { and } P_{10}=\frac{-2 \mu\left[k_{10}\left(n_{02}{ }^{2}-\tau^{2}\right)+\tau^{2} k_{00}\right.}{g_{0}} \text {. }
\end{aligned}
$$

In the above:

$$
\begin{gathered}
g_{0}=\left(n_{02}{ }^{2}-\tau^{2}\right)^{2}+4 k_{00}{ }^{2} \tau^{2}, \\
D_{6}=1+\mu_{L} \frac{\tau^{2}-4 \tau s+2 s^{2}}{\gamma \tau^{2}-2(\gamma+1) \tau s+(\gamma+1) s^{2}},
\end{gathered}
$$

and $n_{02}=\frac{n_{2}}{n_{0}}, k_{00}=\frac{k_{0}}{n_{0}}, k_{01}=\frac{k_{0} k}{n_{0}^{2}}, k_{10}=\frac{k}{n_{0}}$.

Here $\tau=\omega / n_{0}$ and $s=\Omega_{0} / n_{0}$ are dimensionless eigenfrequency of the system and rotor angular speed, respectively.

It follows from the second equation in Eq. (25) that:

$$
g_{1} P_{8}=\mu\left(1-P_{8}\right)+\frac{3 \mu c_{10}}{4}\left(1-P_{8}\right)^{3} B^{2},
$$

where $g_{1}=\left(n_{02}^{2}-\tau^{2}\right)+2 i k_{00} \tau$ and $c_{10}=\frac{c_{1}}{c_{0}}$.

The formulae for $B$ and $D$ can be derived from the last equation and Eq. (29), respectively. They are:

$$
B=\sqrt{\frac{4\left[g_{1} P_{8}-\mu\left(1-P_{8}\right)\right]}{3 \mu c_{10}\left(1-P_{8}\right)^{3}}}
$$

$$
\text { and } D=\left(P_{9}+i P_{10}\right) \sqrt{\frac{4\left[g_{1} P_{8}-\mu\left(1-P_{8}\right)\right]}{3 \mu c_{10}\left(1-P_{8}\right)^{3}}} \text {. }
$$

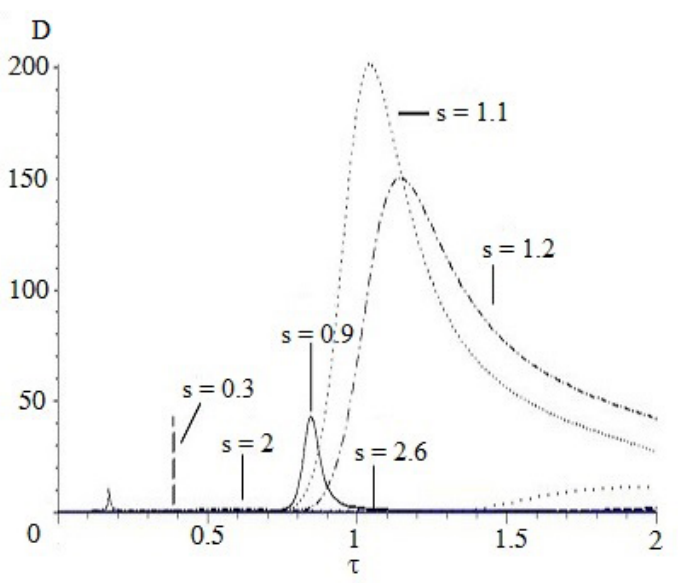

Fig. 3. Foundation amplitude $D=D(\tau, s)$ at $\gamma=15.24$, taking into account damping 


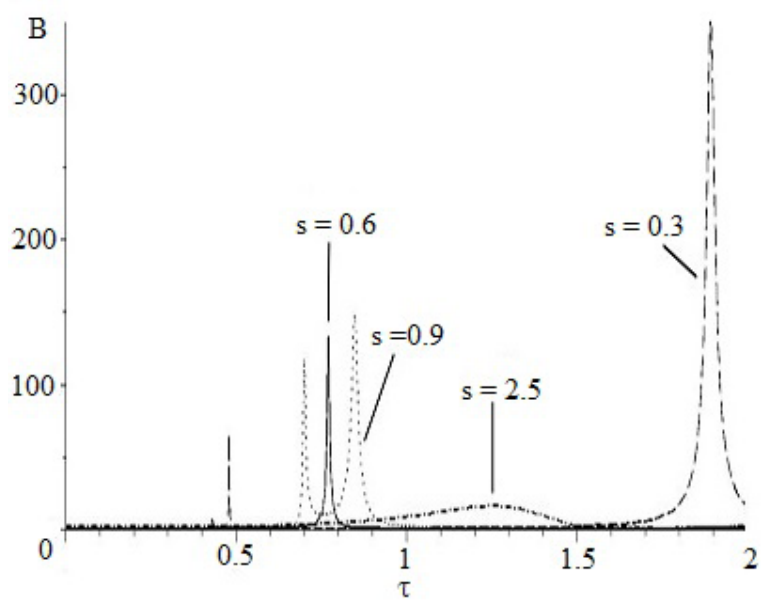

Fig. 4. Rotor amplitude $B=B(\tau, s)$ at $\gamma=4.56$, taking into account damping

Below, we use the same notation for the modulae of these complex-valued quantities, i.e.

$$
\begin{gathered}
B \equiv \sqrt{(\operatorname{Re} B)^{2}+(\operatorname{Im} B)^{2}}, \\
D \equiv \sqrt{(\operatorname{Re} D)^{2}+(\operatorname{Im} D)^{2}} .
\end{gathered}
$$

It is clear that the free vibration amplitudes of the rotor $B$ and the foundation $D$ depend on the vibration frequency $\omega$ of the nonlinear system in question. The formulae above enable calculating amplitude vs frequency graphs at a fixed angular speed of the rotor.

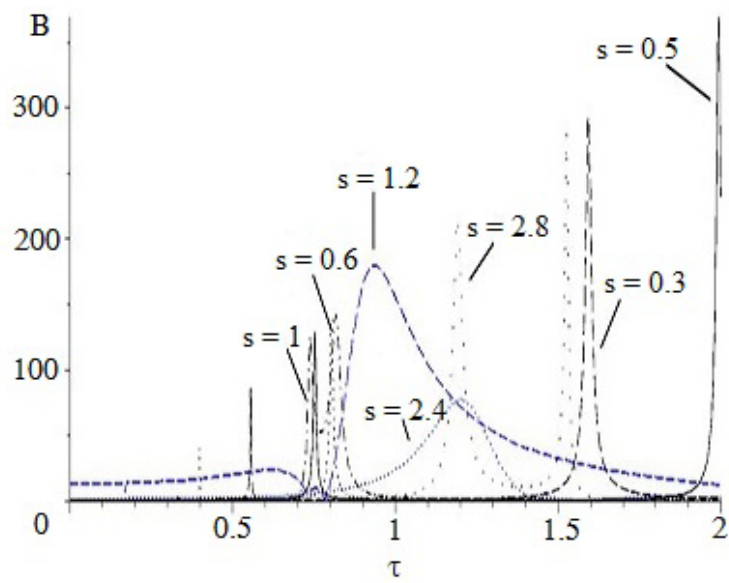

Fig. 6. Rotor amplitude $B=B(\tau, s)$ at $\gamma=2.6$, taking into account damping

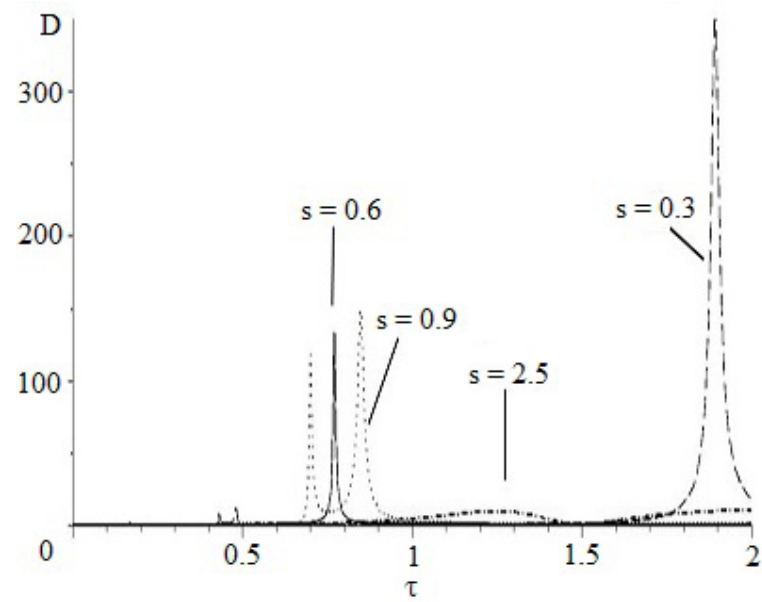

Fig. 5. Foundation amplitude $D=F(\tau, S)$ at $\gamma=4.56$, taking into account damping

\section{NUMERICAL RESULTS}

Let us study the rotor and foundation amplitudes determined in the previous section. In Figs. 2 to 11 graphs demonstrating unstable self-oscillation zones of the rotor $(B)$ and foundation $(D)$ versus the parameters $\tau$ and $s$ are presented for various values of the quantity $\gamma$ characterizing the relative fluid volume in the cavity. A substantial increase in the self-oscillation amplitude, which is bounded due to external damping, is observed.

For a small fluid volume in the rotor cavity $\left(\gamma=15.24\left(r_{0}=0.93 R\right)\right.$ in Figs. 2 and 3$)$ we obtain two zones of unstable self-oscillation of the rotor and foundation. Note that over the angular speed

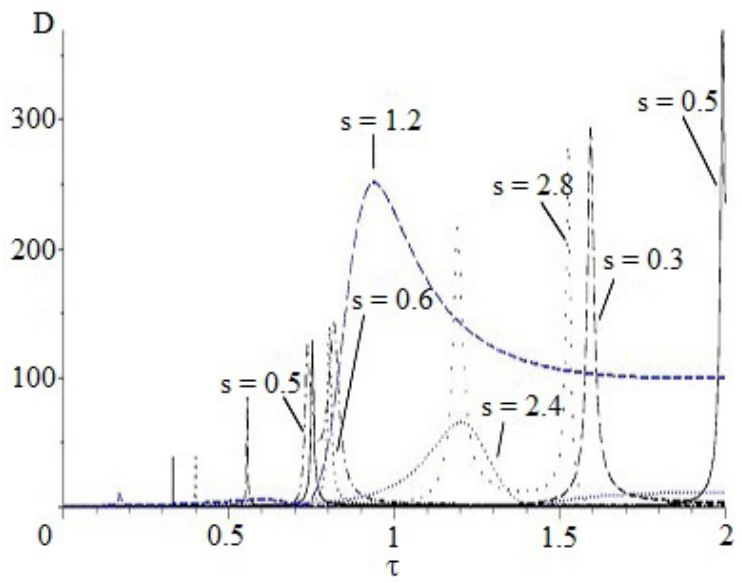

Fig. 7. Foundation amplitude $D=D(\tau, s)$ at $\gamma=2.6$, taking into account damping 
interval $0.3<s<0.9$ the rotor and foundation amplitudes monotonically increase. In this case, the eigenfrequency belongs to the range $0.25<\tau<0.85$. When $s$ varies from 0.9 to 1.1 , the rotor and foundation amplitudes decay quite rapidly, approaching zero values. For the studied fluid volume, third instability zone does not appear over the interval $0.3<s<1$. When the angular speed $s$ increases from 0.5 to 0.85 , the first two zones displace towards greater eigenfrequencies, namely $0.41<\tau<0.85$. Over the interval $0.9<_{S}<1.2$ and in the vicinity of $\tau=0.85$ there is only one instability zone with small self-oscillation amplitudes.

Further increase in the angular speed $(s>1.2)$ results in three instability zones, see Figs. 6 and 7. In these figures rotor and foundation amplitudes are small; in doing so, they first grow slowly and then slowly decay beginning from the value $s=2.8$. In this case, the eigenfrequencies associated with unstable regimes increase in $s$.

The third instability zone gradually disappears as the fluid volume in the cavity increases. For example, at $\gamma=4.56\left(r_{0}=0.8 R\right)$, see Figs. 4 and 5 , the third zone appears at $s>1$. Large rotor B and foundation D amplitudes occur over the interval $0.3<s<1$. In this case, the first instability zone belongs to the intervals $0.3<s<1$ and $0.45<\tau<0.85$, while the second one is over the interval $0.3<s<0.6$. The eigenfrequencies beginning with $\tau=1.4$ gradually decay approaching the value $\tau=0.6$. Next, over the interval $0.6<s<1$, the eigenfrequencies slowly increase. At $s>1.2$ three instability zones occur. However, the related rotor and foundation amplitudes are rather small.

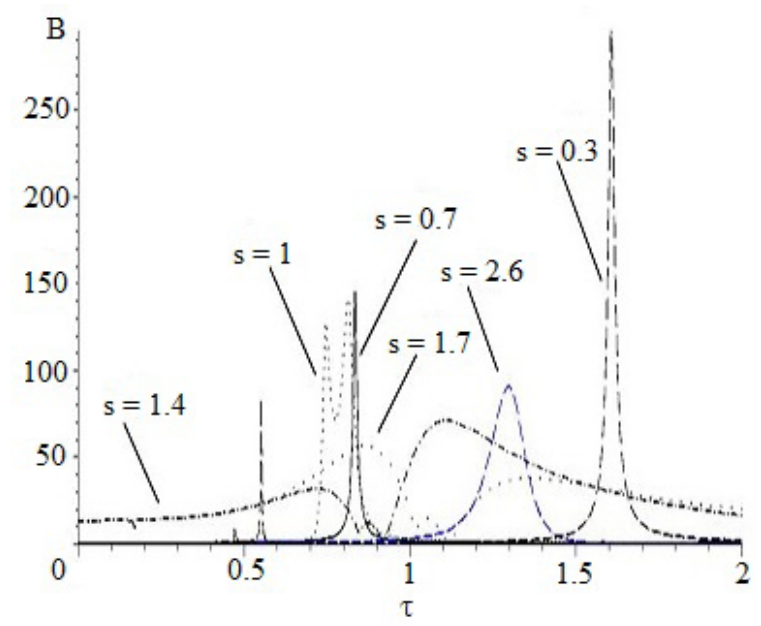

Fig. 8. Rotor amplitude $B=B(\tau, s)$ at $\gamma=1.67$, taking into account damping
In the case of a further increase of the fluid volume in the cavity, only two instability zones remain. In particular, if a third or half of the cavity is fluid filled, i.e. $\gamma=2.6\left(r_{0}=2 R / 3\right)$ in Figs. 6 and 7 , or $\gamma=2.67\left(r_{0}=0.5 R\right)$ in Figs. 8 and 9 , respectively, the system performs large amplitude self-oscillation.

At $\gamma=2.6 \quad\left(r_{0}=2 R / 3\right) \quad$ the self-oscillation amplitudes over the interval $0.3<s<0.5$ grow in $s$, see Figs. 6 and 7. The eigenfrequencies associated with unstable self-oscillation of the rotor and foundation also increase. Over the interval $0.5<s<0.6$ the values of $\tau$ rapidly decay, whereas over the interval $0.6<s<1$ they increase again along with selfoscillation amplitudes; in doing so, the latter are rather significant.

Over the interval $1<_{S}<1.2$ the self-oscillation amplitudes and eigenfrequencies (or critical frequencies) $\tau$ corresponding to rotor and foundation instability zones experience a sharp decay. Over the interval $1.2<_{S}<2.5$ the rotor and foundation perform small amplitude self-oscillation, and critical frequencies slowly grow. At $s>2.5$ the amplitudes sharply increase and the eigenfrequencies also rapidly grow.

The graphs of the rotor and foundation amplitudes $B$ and $D$ versus the dimensionless eigenfrequency $\tau$ are presented in Figs. 8 and 9 for several values of the rotor angular speed $s$. The effect of external damping is incorporated for a half-filled cavity, i.e. at $\gamma=1.67$. Inspection of the graphs shows that the maximal (minimal) values of the amplitudes $B$ and $D$ displace to larger (smaller) $\tau$ as $s$ varies from zero to one. In this

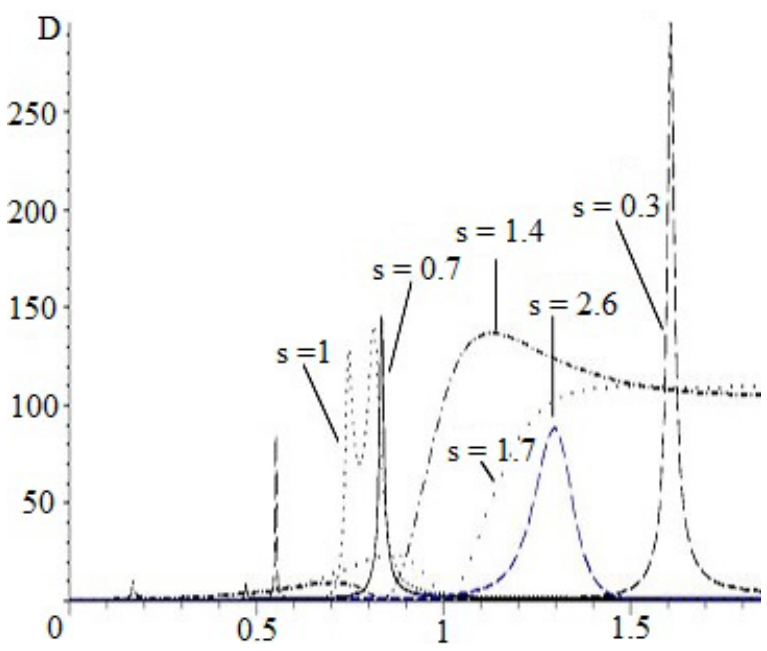

Fig. 9. Foundation amplitude $D=D(\tau, s)$ at $\gamma=1.67$, taking into account damping 


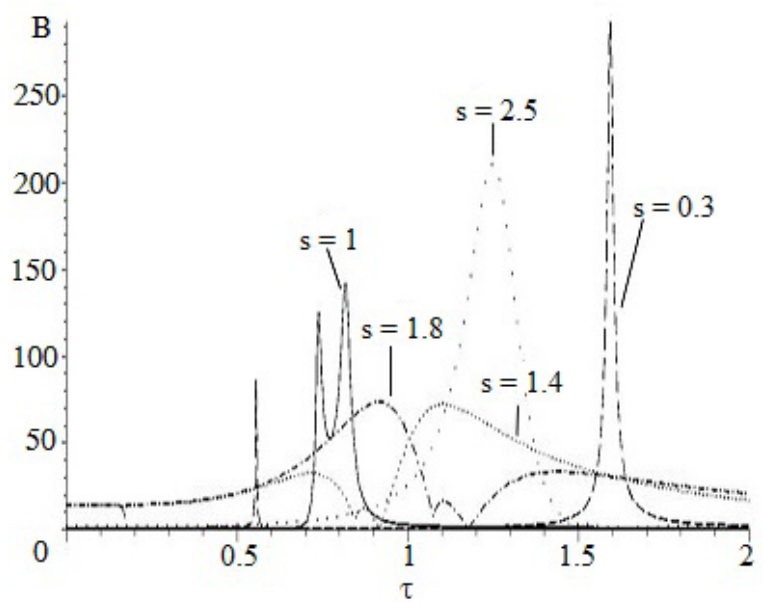

Fig. 10. Rotor amplitude $B=B(\tau, S)$ at $\gamma=1.03$, taking into account damping

case the minima and maxima of $B$ and $D$ lie over the intervals $0.65<\tau<0.85$ and $0.85<\tau<1.8$, respectively.

Over the range $0.6<_{S}<0.85$, the maxima and minima of $B$ and $D$ belong the interval $0.65<\tau<0.85$. Next, when $s$ varies from 1 to 1.4 , the $B$ and $D$ peaks as well as the frequencies $\tau$ decrease. For $s=1.7$ a $B$ maximum is observed at $\tau=0.95$ and its minimum takes place at $\tau=1.2$. In this case, the $D$ peaks have opposite locations. At larger angular speeds, when $s>1.7$, the $B$ and $D$ maxima and minima grow along with the eigenfrequencies $\tau$. For more than a half fluid filled cavity amplitudes and frequencies behave similarly to the consideration above, see Figs. 6 to 9. In the latter case, self-oscillation amplitudes are lower than before. The second instability zone

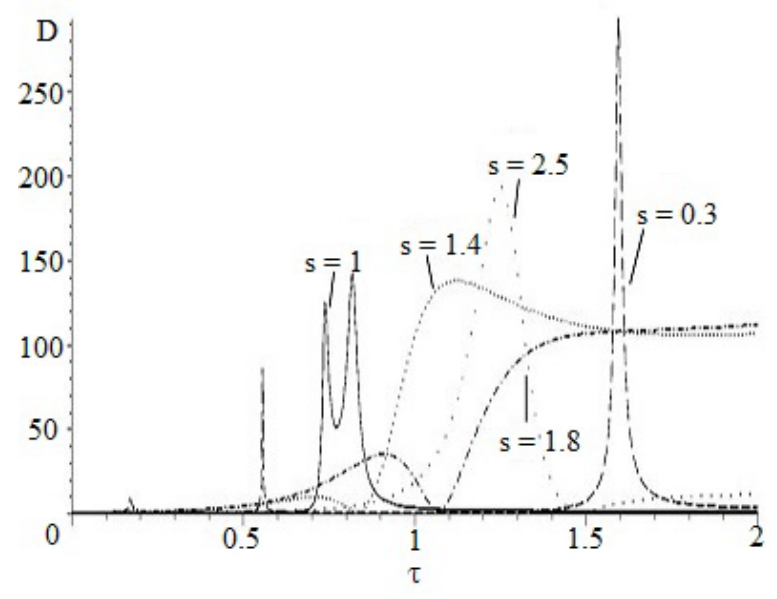

Fig. 11. Foundation amplitude $D=D(\tau, s)$ at $\gamma=1.03$, taking into account damping

disappears at large fluid volumes in the cavity. At a very large relative volume corresponding to the value $\gamma=1.03\left(r_{0}=1 R / 8\right)$ rotor and foundation selfoscillation amplitudes related to the first instability, zone do not vary over a wide range of rotor angular speeds $(0.3<s<1$ and $s>1.8)$, see Figs. 10 and 11. In this case, the eigenfrequency is $\tau=0.85$. The second instability zone arises over the intervals $0.3<s<0.5$ and $1.4<s<1.8$. It is characterized by small rotor and foundation self-oscillation amplitudes. Thus, selfoscillation amplitudes are not that significant for small fluid volumes in the cavity.

As the fluid volume approaches a third or half of the cavity, self-oscillation amplitudes take relatively large values, both for small and large angular speeds.

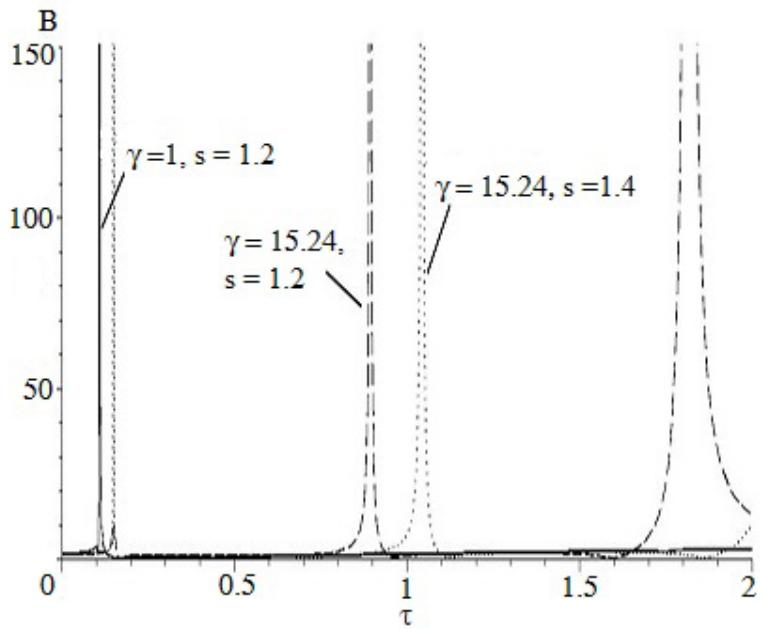

Fig. 12. Rotor skeleton curves versus $s$ and $\gamma$

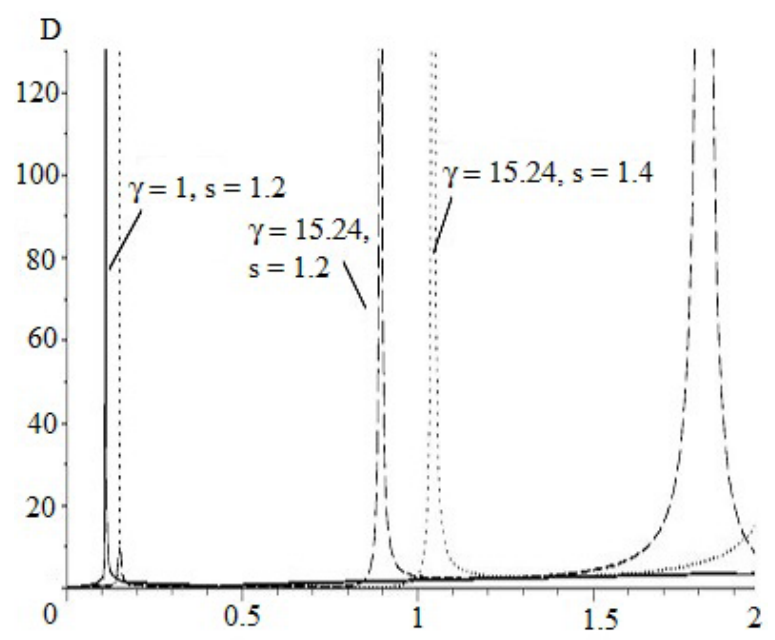

Fig. 13. Foundation skeleton curves for several values of $s$ and $\gamma$ 


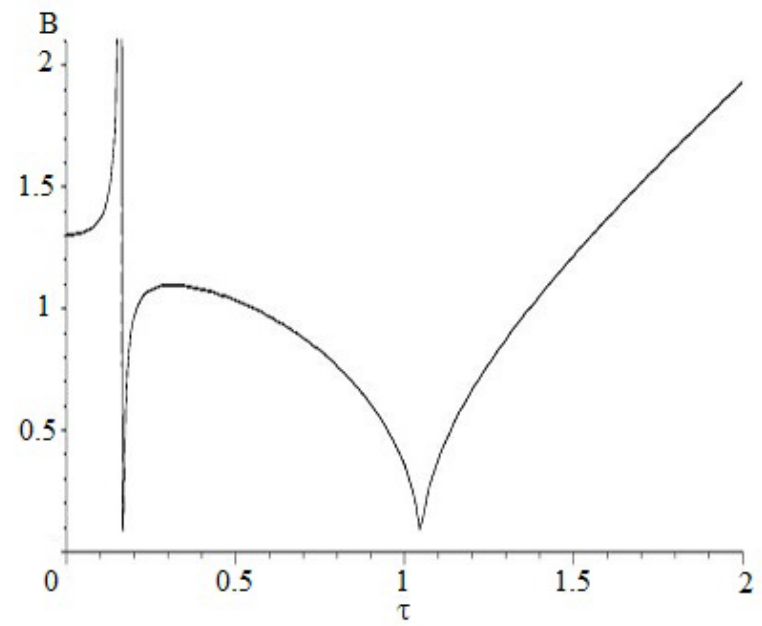

Fig. 14. Rotor skeleton curve neglecting effect of fluid

For a rotor cavity containing significant fluid volumes, there is a single instability zone characterized by the constant amplitude and eigenfrequency. The skeleton curves $B$ and $D$ of the rotor and foundation are shown in Figs. 12 and 13, taking into consideration the presence of fluid in the cavity. These figures display three critical values of $\tau$ corresponding to vertical asymptotes of skeleton curves. In the general case, they exist at any value of $\mathrm{s}$ over the whole range of the eigenfrequencies $\tau$. The rotor and foundation amplitudes tend to infinity at the critical values, i.e. $B \rightarrow \infty, D \rightarrow \infty$. It is clearly seen that the first critical frequency lies in the vicinity of $\tau=0.11$. It virtually does not depend on the variation of the angular speed $s$ and is very weakly dependent of the relative fluid volume $\gamma$. This frequency is slightly displaced towards greater values of $\tau$ as the relative fluid volume grows, i.e. $\gamma \rightarrow 1$. In this case, $\tau \leq 0.14$. The second critical frequency grows in $s$ for any fluid volume.

At the same time its increase $(\gamma \rightarrow 1)$ leads to a decrease of the second critical frequency. The third critical frequency mainly arises at low rotor angular speeds and quite small fluid volumes as well as at high angular speeds and large fluid volumes. The third critical frequency disappears when the fluid volume decreases, i.e. at $\gamma>1.03$ and $s>1$ or at $\gamma<15.24$ and $s<1.4$.

The increase of the rotor and foundation amplitudes $B$ and $D$ at a slow variation of the eigenfrequency $\tau$ below critical frequencies is very specific for all skeleton curves. After the passages through critical frequencies rotor and foundation amplitudes sharply decay over a narrow range of $\tau$. Further increase in $\tau$ results in the growth of the

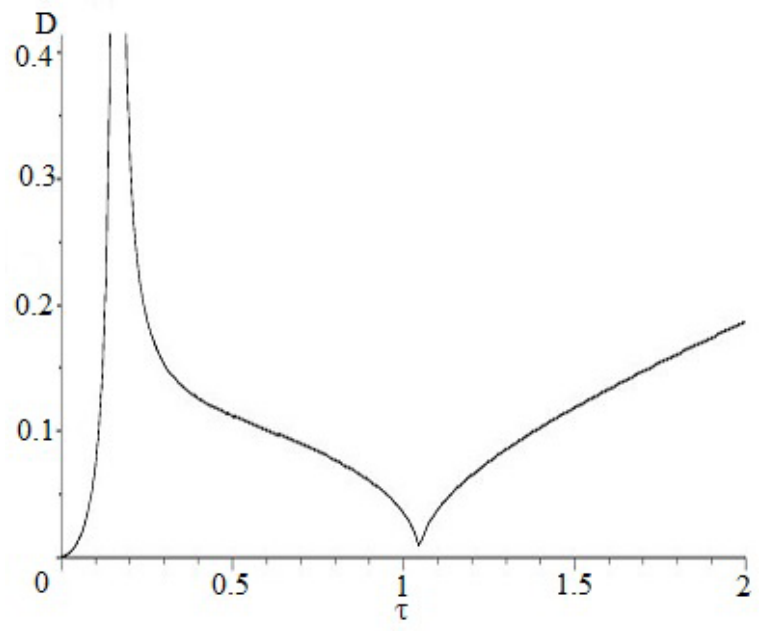

Fig. 15. Foundation skeleton curve neglecting effect of fluid

amplitudes $B$ and $D$. For the rotor and foundation skeleton curves incorporating the effect of fluid, we obtain $\omega=\sqrt{\frac{2 c_{2}}{M\left(1+\mu D_{6}\right)}}$ with the vertical asymptote $\tau=n_{02} \sqrt{\frac{1}{1+\mu D_{6}}}$.

The occurrence of two (or three in the general case) peaks of rotor and foundation amplitudes (damping is incorporated) and of skeleton curves (damping is ignored) at any value of the parameters $s$ and $\gamma$ is caused by fluid vibrations along a free surface inside the rotor cavity. The graphs in Figs. 14 and 15, displaying rotor and foundation skeleton curves, illustrate the last observation.

For synchronous precession, i.e. at $\tau=s$, the rotor and foundation amplitudes do not depend on the fluid volume. In this case, they follow from Eqs. (30) and (31) with $D_{6}=1+\mu_{L}$.

\section{SPECIAL CASES}

The analytical solution obtained in the paper enables an immediate qualitative insight into practically important special setups. Below we study the limiting case of an empty rotor and also address the widely disputed phenomenon of self-centring. Consider first an empty rotor. In this case, the fluid force is zero, i.e. $F_{r}=0$. Free vibrations are specified by the coordinates:

$$
x=a \cos \omega t \quad \text { and } \quad x_{1}=b \cos \omega t,
$$

where $a, b$ are amplitudes and $\omega$ is eigenfrequency. 
Using the previous notations in the absence of imbalance in Eq. (25) and taking into account Eq. (32), we find rotor and foundation amplitudes, respectively:

$$
\begin{aligned}
& a^{2}=\frac{4\left[\omega^{2}\left(n_{2}^{2}-\omega^{2}\right)-n_{0}^{2}\left(n_{2}^{2}-(1+\mu) \omega^{2}\right)\right]\left(n_{2}^{2}-\omega^{2}\right)^{2}}{3 n_{1}\left(n_{2}^{2}-(1+\mu) \omega^{2}\right)^{3}}, \\
& b^{2}=\frac{4 \omega^{4} \mu^{2}\left[\omega^{2}\left(n_{2}^{2}-\omega^{2}\right)-n_{0}^{2}\left(n_{2}^{2}-(1+\mu) \omega^{2}\right)\right]}{3 n_{1}\left(n_{2}^{2}-(1+\mu) \omega^{2}\right)^{3}},
\end{aligned}
$$

From the Eqs. (33) and (34) we can determine the dependence of the amplitudes on the frequency $\omega$. The graphs of $a$ and $b$ versus $\omega$ are key curves characterizing the system. From the last formulae, it is clear that for $\omega \rightarrow \sqrt{2 c_{2} /(m+M)}$ the amplitudes of $a$ and $b$ tend to infinity, i.e. a resonance occurs. The rotor amplitude $a$ is equal to zero when the frequency $\omega$ is equal to $\omega=\sqrt{2 c_{2} / M}$. In this case, the squared foundation amplitude becomes $b^{2}=-4 n_{0}^{2} /\left(3 n_{1}\right)$.

It is clear from the graphs in Figs 14 and 15 that rotor and foundation amplitudes grow over the interval $0<\tau<0.18$ approaching the vertical asymptote $\tau=0.18$ associated with the critical value of the eigenfrequency. Next, over the interval $0.18<\tau<0.25$ the rotor amplitude starts growing as $\tau$ increases. Then it slowly decays, approaching zero at $\tau=1.15$. At larger values of $\tau$ the amplitude increases along a parabola branch. The foundation skeleton curve has a similar shape. However, it also demonstrates a few peculiarities. In particular, it begins at the origin and grows approaching the asymptote $\tau=0.18$. At larger $\tau$ it slowly decays approaching zero at $\tau=1.15$. Finally, it grows along a parabola branch.

Forced vibrations are caused by an imbalance of the system. For the sake of simplicity, we insert a phase angle $\varepsilon$ into the expression for the force $m e \Omega_{0}^{2} \cos \left(\Omega_{0} t+\varepsilon\right)$. The nonlinear forced vibrations of an empty rotor are governed by:

$$
\begin{gathered}
\ddot{x}+n_{0}^{2}\left(x-x_{1}\right)+n_{1}\left(x-x_{1}\right)^{3}+2 k \dot{x}= \\
=e \Omega_{0}^{2} \cos \left(\Omega_{0} t+\varepsilon\right), \\
\ddot{x}_{1}+n_{2}^{2} x_{1}-n_{01}^{2}\left(x-x_{1}\right)-n_{10}\left(x-x_{1}\right)^{3}=0 .
\end{gathered}
$$

On substituting the formulae:

$$
x=a_{1} \cos \Omega_{0} t \quad \text { and } \quad x_{1}=b_{1} \cos \Omega_{0} t .
$$

Into Eq. (35) we finally get:

$$
\begin{gathered}
\left(b_{1}\right)_{1,2}=\frac{ \pm \Omega_{0} \sqrt{\mu^{2} e^{2} \Omega_{0}^{2}-4 k_{1}^{2} a_{1}^{2}}+\mu \Omega_{0}^{2} a_{1}}{n_{2}^{2}-\Omega_{0}^{2}}, \\
\operatorname{tg} \varepsilon=\frac{2 k a_{1} \Omega_{0}}{\left(n_{0}^{2}-\Omega_{0}^{2}\right) a_{1}-n_{0}^{2} b_{1}+\frac{3}{4} n_{1}\left(a_{1}-b_{1}\right)^{3}} \\
\frac{\left(n_{2}^{2}-n_{01}^{2}-\Omega_{0}^{2}\right)\left( \pm \Omega_{0} \sqrt{\mu^{2} e^{2} \Omega_{0}^{2}-4 k_{1}^{2} a_{1}^{2}}+\mu \Omega_{0}^{2} a_{1}\right)}{n_{2}^{2}-\Omega_{0}^{2}}- \\
-n_{01}^{2} a_{1}-\frac{3}{4} n_{10}\left(a_{1}-\frac{ \pm \Omega_{0} \sqrt{\mu^{2} e^{2} \Omega_{0}^{2}-4 k_{1}^{2} a_{1}^{2}}+\mu \Omega_{0}^{2} a_{1}}{n_{2}^{2}-\Omega_{0}^{2}}\right)^{3}=0 .
\end{gathered}
$$

The Eq. (38) determines an amplitude-frequency characteristic of the rotor motion along $O x$ axis; a similar formula holds for $O y$ axis. This equation can be transformed to a sixth order polynomial in $a_{1}$. We also remark that for $n_{10}>0$ the system is rigid, whereas for $n_{10}<0$ it is elastic.

The studied nonlinear system supports multiple periodic regimes for the angular speed $\Omega_{0}$ varying within certain limits. In this case, the vertical lines $\Omega_{0}=n_{2}$ are the asymptotes of the amplitude-frequency characteristic. On introducing the value of the rotor amplitude $a_{1}$ into Eq. (37), we may derive a formula for $b_{1}\left(\Omega_{0}\right)$ expressing the amplitude-frequency characteristic of the foundation.

Next, the issue related to self-centering and pressure on support will be discussed. The formulae in Eqs. (37) and (38) enable to find limiting values of the amplitudes $a_{1}$ and $b_{1}$, i.e. $\lim _{\Omega_{0} \rightarrow \infty} a_{1}=\mp e$, and $\lim _{\Omega_{0} \rightarrow \infty} b_{1}=-\left(a_{1} \pm e\right) \mu=0$. In particular, for regular precession we get $\lim _{\Omega_{0} \rightarrow \infty} a_{1}=-e, \lim _{\Omega_{0} \rightarrow \infty} b_{1}=0$. Hence, at an infinite increase of the angular speed the vector associated with static imbalance is directed towards the origin and tends to displace the rotation axis towards the vertical axis $\mathrm{Oz}$. In the latter case, a static imbalance of the rotor has no effect. The observed self-centering of a rigid rotor on rolling bearings in the presence of an elastic foundation is an important feature of the system.

The pressure along the contact zone of the rotor and foundation can be evaluated by calculating the reaction forces at bearing supports. Let us replace the action of the rotor by its reaction forces. As each bearing support is subject to the same reaction force, the rotor performs the plane-parallel motion. The equation of foundation motion is then given by $M \ddot{x}_{1}+2 c_{2} x_{1}=2 R_{1 x}$, resulting in $R_{1 x}=b_{1}\left(c_{2}-M \Omega_{0}^{2} / 2\right)$, which means that at $M=2 c_{2} / \Omega_{0}^{2}$ the radial pressure along the contact of the rotor and foundation is zero. 
Therefore, for a constant operating speed, we may define a stiffness coefficient of the elastic foundation for which the aforementioned pressure is zero. The foundation reaction force acting on the supports is $R_{2}=c_{2} b_{1}$, where $b_{1}$ is the foundation amplitude. It tends to zero at an infinite increase in the angular speed $\Omega_{0}$, i.e. in the case of self-centering.

\section{CONCLUDING REMARKS}

A generalized dynamic model of a rotor-fluidfoundation system taking into account the nonlinear stiffness of rolling bearings along with fluid and foundation vibrations is developed and investigated.

The obtained results enable optimising the parameters of the rotor, foundation, and fluid and are of interest for reducing stresses along the contact surfaces, forced vibrations amplitudes as well as the width of instability zones. There is also room for adapting the conditions supporting self-centering of the system.

Suppression of harmful rotor vibrations by choosing appropriate system parameters, including foundation deformability, is economically efficient and has the potential to be implemented in technology. In this case, an elastic foundation plays a role of a sort of counterbalance.

\section{REFERENCES}

[1] Tondl, A. (1965). Some Problems of Rotor Dynamics. Chapman and Hall, London.

[2] Penny, J., Friswell, M., Garvey, S., Lees, A. (2010). Dynamics of Rotating Machines. Cambridge University Press, Cambridge.

[3] Vance, J.M., Zeidan, F.Y., Murphy, B. (2010). Machinery Vibration and Rotor Dynamics. Wiley, Hoboken, DOI:10.1002/9780470903704.

[4] Bolotin, V.V. (1963). Nonconservative Problems of the Theory of Elastic Stability. Macmillan, New York.

[5] Derendyayev, N.V., Soldatov, I.N., Vostrukhov, A.V. (2006). Stability and Andronov-Hopf bifurcation of steady-state motion of rotor system partly filled with liquid: Continuous and discrete models. Journal of Applied Mechanics, vol. 73, no. 4, p. 580-589, DOl:10.1115/1.2164514.

[6] Rahimov, E.R., Rahmatullaev, A.Sh., Kydyrbekuly, A.B. (1987). Dynamics of unbalanced rotor in the interaction with the other physical fields. Transactions of the $7^{\text {th }}$ World Congress on the Theory of Machines and Mechanisms, Sevilla, p. 532-539.

[7] Manasseh, R. (1994). Distortions of inertia waves in a rotating fluid cylinder forced near its fundamental mode resonance. Journal of Fluid Mechanics, vol. 265, p. 345-370, DOI:10.1017/S0022112094000868.

[8] Changsheng, Z. (2006). Experimental investigation into the effect of fluid viscosity on instability of an overhung flexible rotor partially filled with fluid. Journal of
Vibrations and Acoustics, vol. 128, no. 3, p. 392-401, DOI:10.1115/1.2166857.

[9] Lichtenberg, G. (1980). Experimentell Untersuchung des Schurngugsverhaltens eines flussig - keitsgefullten Rotors mit Kreiselwirkung. VDI-Berichte, no. 381, p. 161-166.

[10] Urbiola-Soto, L., Lopez-Parra, M. (2013) Liquid self-balancing device effects on flexible rotor stability. Shock and Vibration, vol. 20, no. 1, p. 109-121, D0l:10.3233/SAV-2012-0731.

[11] Changsheng, Z. (2001). Stability behaviour of an isotropic rotor system anisotropically mounted and partially filled with inviscous fluid. Proceedings of IMAC-19, A Conference on Structural Dynamics, p. 1278-1283.

[12] Kydyrbekuly, A.B. (2012). Rotor-Liquid-Fundament System's Oscillation. Advances in Mechanisms Design. Springer Science+Business Media, Dordrecht, p. 223-229, DOl:10.1007/978-94-007-5125-5_30.

[13] Silin, R., Royzman, V. (1999). The research into the automatic balancing process of rotors with vertical axis of rotation. 10th World Congress on the Theory of Machine and Mechanisms, Oulu, vol. 4. p. 1734-1739.

[14] Childs, D. (1993). Turbomachinery rotor dynamics phenomena. Modeling and Analysis, Wiley-Interscience, New York.

[15] Zebin, Y., Dawei, D., Haiyu, G., Xiaodong, S., Rong, F., Huangqiu, Z. (2015). Rotor mass eccentricity vibration compensation control in bearingless induction motor. Advances in Mechanical Engineering. Article ID 168428, DOI:10.1155/2014/168428.

[16] Savin, L., Solomin, O., Ustinov, D. (1999). Rotor dynamics on friction bearings with cryogenic lubrication. 10th World Congress on the Theory of Machine and Mechanisms, Oulu, vol. 4, p. 1716-1721.

[17] Harsha, S.P. (2005). Non-linear dynamic response of a balanced rotor supported on rolling element bearings. Mechanical Systems and Signal Processing, vol. 19, no. 3, p. 551-578, D0I:10.1016/J.ymssp.2004.04.002.

[18] Isayuk-Sayevskaya, A.R., Kelzon, A.S. (1995). Dynamics of a high-revolution scroll centrifuge. Machine Vibration, vol. 3, no. 4, p. 233-237.

[19] Krämer, E. (1993). Dynamics of Rotors and Foundations. Springer-Verlag, New York, D0I:10.1007/978-3-662-02798-1.

[20] Feng, N., Hahn, E. (1997). Vibration analysis of rotor-bearingpedestal system. Proceedings of the $5^{\text {th }}$ International Congress on Sound and Vibration, Adelaide, p. 2457-2464.

[21] Feng, N., Hahn E. (1998). Experimental identification of the pedestal in a rotor-bearing-pedestal system. Proceedings of the $5^{\text {th }}$ IFTOMM International Conference on Rotor Dynamics, p. 734-745.

[22] Sun, L. (1995). Active Vibration Control of Rotor-Bearing System. PhD Thesis, University of Melbourn, Melbourn.

[23] Bai, C., Zhang, H., Xu, Q. (2010). Experimental and numerical studies on nonlinear dynamic behavior of rotor system supported by ball bearings. Journal of Engineering for Gas Turbine and Power, vol. 132, no. 8, paper 082502, DOl:10.1115/1.4000586.

[24] Aktürk, N., Uneeb, M., Gohar, R. (1997). The effects of number of balls and preload on vibrations associated with 
ball bearings. Journal of Tribology, vol. 119, no. 4, p. 747-753, DOI:10.1115/1.2833880.

[25] Guida, D., Pappalardo, C.M. (2009). Sommerfeld and mass parameter identification of lubricated journal bearings. Applied and Theoretical Mechanics, vol. 4, no. 4, p. 205-214.

[26] Tiwari, M., Gupta, K., Prakash, 0. (2000). Effect of radial internal clearance of a ball bearing on the dynamics of a balanced horizontal rotor. Journal of Sound and Vibration, vol. 238, no. 5, p. 723-756, DOl:10.1006/jsvi.1999.3109.

[27] Tiwari, M., Gupta, K., Prakash, O. (2000) Dynamic response of an unbalanced rotor supported on ball bearings. Journal of Sound and Vibration, vol. 238, no. 5, p. 757-779, DOl:10.1006/jsvi.1999.3109.
[28] Harsha, S.P. (2006). Rolling bearing vibrations - the effects of surface waviness and radial internal clearance. International Journal for Computational Methods in Engineering Science and Mechanics, vol. 7, no. 2, p. 91-111, DOI:10.1080/155022891010015.

[29] Harsha, S.P. (2006). Nonlinear dynamic analysis of a highspeed rotor supported by rolling element bearings. Journal of Sound and Vibration, vol. 290, no. 1-2, p. 65-100, DOl:10.1016/j.jsv.2005.03.008.

[30] Jang, G.H., Jeong, S.W. (2003). Analysis of a ball bearing with waviness considering the centrifugal force and gyroscopic moment of the ball. Journal of Tribology, vol. 125, no. 3, p. 487-498, D0I:10.1115/1.1538618. 